Manuscript submitted for review to

Thin Solid Films

\title{
High quality ZnO:Al transparent conducting oxide films synthesized by pulsed filtered cathodic arc deposition
}

\author{
André Anders ${ }^{\mathrm{a}}$, Sunnie H.N. Lim ${ }^{\mathrm{a}, \mathrm{b}}$, Kin Man Yu ${ }^{\mathrm{a}}$, Joakim Andersson ${ }^{\mathrm{a}, \mathrm{c}}$, Johanna Rosén ${ }^{\mathrm{a}, \mathrm{d}}$; \\ Mike McFarland ${ }^{e}$, and Jeff Brown ${ }^{e}$ \\ ${ }^{a}$ Lawrence Berkeley National Laboratory, Berkeley, California \\ ${ }^{\mathrm{b}}$ University of Sydney, School of Applied Physics, Sydney, Australia \\ c Ångström Laboratory, Uppsala University, Uppsala, Sweden \\ ${ }^{\mathrm{d}}$ Linköping University, Linköping, Sweden \\ ${ }^{\mathrm{e}}$ Acree Technologies Inc., Concord, California
}

April 24, 2009

\begin{abstract}
DISCLAIMER
This document was prepared as an account of work sponsored by the United States Government. While this document is believed to contain correct information, neither the United States Government nor any agency thereof, nor The Regents of the University of California, nor any of their employees, makes any warranty, express or implied, or assumes any legal responsibility for the accuracy, completeness, or usefulness of any information, apparatus, product, or process disclosed, or represents that its use would not infringe privately owned rights. Reference herein to any specific commercial product, process, or service by its trade name, trademark, manufacturer, or otherwise, does not necessarily constitute or imply its endorsement, recommendation, or favoring by the United States Government or any agency thereof, or The Regents of the University of California. The views and opinions of authors expressed herein do not necessarily state or reflect those of the United States Government or any agency thereof or The Regents of the University of California.
\end{abstract}

\section{ACKNOWLEDGMENTS}

This work was supported by the Assistant Secretary for Energy Efficiency and Renewable Energy, Office of Building Technology, and by the Director, Office of Science, Office of Basic Energy Sciences, Division of Materials Sciences and Engineering of the U.S. Department of Energy under Contract No. DE-AC02-05CH11231. K.M. Yu thanks RoseStreet Labs Energy, Inc. for additional support; S.H.N. Lim gratefully acknowledges the financial support by the Australian Research Council (ARC project DP0666883) and the Australian Institute of Nuclear Science and Engineering (AINSE grants AINGRA07012P and AINGRA07094); J. Rosén and J. Andersson acknowledge support by the SSF Strategic Research Centre on Materials Science for Nanoscale Surface Engineering, and the Wenner-Gren Foundations (both Sweden), respectively. 


\title{
High quality ZnO:Al transparent conducting oxide films synthesized by
} pulsed filtered cathodic arc deposition

André Anders a, Sunnie H.N. Lim ${ }^{\mathrm{a}, \mathrm{b}}$, Kin Man Yư ${ }^{\mathrm{a}}$, Joakim Andersson ${ }^{\mathrm{a}, \mathrm{c}}$, Johanna Rosén ${ }^{\mathrm{a}, \mathrm{d}}$;

Mike McFarland ${ }^{\mathrm{e}}$, and Jeff Brown ${ }^{\mathrm{e}}$

${ }^{\text {a }}$ Lawrence Berkeley National Laboratory, Berkeley, California

${ }^{\mathrm{b}}$ University of Sydney, School of Applied Physics, Sydney, Australia

c Ångström Laboratory, Uppsala University, Uppsala, Sweden

d Linköping University, Linköping, Sweden

${ }^{\mathrm{e}}$ Acree Technologies Inc., Concord, California

\begin{abstract}
Aluminum-doped zinc oxide, $\mathrm{ZnO}: \mathrm{Al}$ or $\mathrm{AZO}$, is a well-known n-type transparent conducting oxide with great potential in a number of applications currently dominated by indium tin oxide (ITO). In this study, the optical and electrical properties of AZO thin films deposited on glass and silicon by pulsed filtered cathodic arc deposition are systematically studied. In contrast to magnetron sputtering, this technique does not produce energetic negative ions, and therefore ion damage can be minimized. The quality of the AZO films strongly depends on the growth temperature while only marginal improvements are obtained with post-deposition annealing. The best films, grown at a temperature of about $200^{\circ} \mathrm{C}$, have resistivities in the low to mid $10^{-4}$ $\Omega \mathrm{cm}$ range with a transmittance better than $85 \%$ in the visible part of the spectrum. It is remarkable that relatively good films of small thickness $(60 \mathrm{~nm})$ can be fabricated using this method.
\end{abstract}

Keywords: transparent conducting oxide, aluminum-doped zinc oxide, filtered cathodic arc 


\section{Introduction}

Doped zinc oxide, and in particular aluminum doped zinc oxide ( $\mathrm{ZnO}: \mathrm{Al}$ or $\mathrm{AZO})$, has become a much investigated and used transparent conducting material that competes with the more established indium tin oxide (ITO). While ITO remains superior in its combination of high transmittance, low resistivity, and good stability, the greater abundance of the constituents and the lower cost of AZO makes it one of the preferred substitutions, especially as the optical and electrical parameters are comparable to ITO. Much has been written about transparent conducting oxides (TCOs) such as ITO and AZO due to the tremendous importance in applications like displays, photovoltaic cells, touchscreens and electrochromic windows, and therefore we refer to the vast literature for further background information, e.g. [1-10].

Magnetron sputtering is a preferred deposition technique for TCOs because of its ease of use and scalability. The energetics of film growth by magnetron sputtering is rather complicated because the flux of particles arriving at the substrate contain mostly atoms of lower energy $(\sim \mathrm{eV})$, gas ions of just a few $\mathrm{eV}$, and some negative ions of much higher energy, typically a few $100 \mathrm{eV}$ [11]. The terms "low" and "high" particle energy should be understood in relation to the energies relevant for film growth. For "energetic condensation", critical energies are the displacement energy and the surface binding energy. The energy of the negative ions can be much higher than those characteristic energies of the solid, and therefore negative ions can create significant "ion damage" to the crystalline grains of the growing film. As a result, one can expect inferior optical and electrical properties caused by ion damage, as evidenced by the very non-uniform properties of stationary substrates placed in front of a magnetron target $[6,12,13]$. The origin of the high energy of negative ions (up to the full target potential of several $100 \mathrm{eV}$ $[11,14])$ moving away from the target is related to the near-target electric field - the same field 
that accelerates positive ions toward the target thereby producing sputtered atoms and secondary electrons. The issue is therefore fundamental to magnetron sputtering but does not exist for filtered cathodic arc deposition because the potential difference, causing an accelerating field, is much smaller (typically $\sim 20 \mathrm{~V}$ only).

In addition to bombardment by energetic negative ions, the substrate is also subject to bombardment by positive ions and neutral atoms. In magnetron sputtering, this contribution can be quite important because, while most sputtered atoms have a kinetic energy of $\sim 1-5 \mathrm{eV}$, the distribution exhibits a long tail reaching all the way up to about $100 \mathrm{eV}$ [15]. Additionally, sputtering (primary) ions may be reflected and become neutrals that enter the substrate region with high energy. All of those processes do not apply to cathodic arc deposition.

In this contribution we investigate the properties of AZO grown by pulsed filtered cathodic arc deposition [16] in anticipation that good film material can be grown due to the absence of the large potential difference. The cathodic arc technique, not even mentioned in some reviews on AZO [9, 17], provides an interesting range of energy of the condensing, filmforming particles. The average kinetic energy of zinc ions for the vacuum case is $36 \mathrm{eV}$ [18] with significant amounts of ions in the tail of the energy distribution function up to 1.5 of the average value [19]. Collisions of zinc ions with processing gas (oxygen) will reduce the zinc kinetic energy and at the same time provide excitation, ionization, and kinetic energy to the gas. This has been investigated for other cathodic arc - gas interactions, e.g. [20, 21]. Additionally, the dense zinc arc plasma also causes excitation and ionization of oxygen, and therefore the deposition process is "energetic" [22-24] with contributions of both kinetic and potential energy to the film growth process $[16,25]$. Previous work using DC filtered arcs focused on room 
temperature or near-room-temperature deposition $[10,26,27]$, whereas we will show that elevated temperature growth is very beneficial for the electrical and optical properties.

We will briefly describe the pulsed arc deposition process, characterize the films, and provide correlations of process parameters to thin film properties. The focus of this investigation is on relatively thin films of $150 \mathrm{~nm}$ or less because such thin films are of great technical interest but generally show less favorable electrical properties than thicker films.

\section{Experimental}

Thin films $(\sim 100 \mathrm{~nm})$ of AZO were deposited using two similar setups as shown in Fig. 1. The plasma source was a "minigun" [28] with a rod cathode of $6.25 \mathrm{~mm}$ (1/4 inch) diameter; the cathode was enclosed by an alumina ceramic such that the cathode spots can operate only on the front surface of the rod. The cathode material was zinc doped with various levels of aluminum (0-6 at $\%)$. The zinc-aluminum plasma stream passed a $90^{\circ}$-bent open filter [16] to remove most of the macroparticles. Pure oxygen was injected into the chamber using a mass flow controller (MKS Instruments); the gas contamination level was dominated by the residual water vapor of the high vacuum chamber. The process gas composition was monitored by a differentially pumped quadrupole mass spectrometer (model RGA100 by SRS). The main chamber was cryogenically pumped to a base pressure of about $1 \times 10^{-4} \mathrm{~Pa}$. The maximum pumping speed of the cryogenic pump of $1500 \mathrm{l} / \mathrm{s}$ could be reduced through an adjustable gate valve (VAT); the combination of flow and pumping speed resulted in a total pressure monitored by a Baratron ${ }^{\circledR}$ capacitance manometer. 
Arc pulses of $1 \mathrm{~ms}$ duration and amplitude of 780 A were delivered with a repetition rate of usually 3 to 4 pulses per second by a pulse-forming network [29]. The arc current was measured using a $0.01 \mathrm{~V} / \mathrm{A}$ Pearson coil and recorded on a digital oscilloscope (Tektronix).

Microscope glass slides and pieces of silicon wafers, some with native oxide and others with a $500 \mathrm{~nm}$ thick layer of $\mathrm{SiO}_{2}$, were mounted on a slowly rotating holder and heated from room temperature up to $400^{\circ} \mathrm{C}$ on the growth side using a 4-lamp radiative heater, as indicated in Fig. 1. In one series of depositions without rotation, the substrate holder temperature was measured with a K-type thermocouple, and in another series (with rotation) the substrate surface temperature was measured through a quartz window using an infrared Raytek ${ }^{\circledR}$ temperature sensor. A systematic error of about $50 \mathrm{~K}$ due to reflection of radiation from the heater was determined, and the indicated temperature was corrected accordingly. The largest error of the surface temperature is due to estimates of the emissivity, and in fact the expected drop of the indicated temperature during growth was seen as the result of a reduction of the emissivity as the AZO films grew. The here reported temperatures are values corrected for systematic errors.

Film thickness was routinely measured using step profilometry (Dektak IIA) and further cross-checked by Rutherford backscattering spectrometry (RBS) using a $2 \mathrm{MeV}$ helium ion beam. Electrical properties (sheet resistance, resistivity, electron concentration and mobility) of the films were characterized by four point probe and Hall effect measurements in the Van der Pauw geometry using an Ecopia HMS3000 system. Transmittance and absorption edge of AZO films were determined by photospectrometry in the wavelength range of 200-2500 nm (PerkinElmer Lambda 19 and 950). Structural and compositional information on AZO films were obtained by RBS and X-ray diffraction (XRD). Microstructural information on selected samples was obtained by high resolution transmission electron microscopy (TEM: JEOL 300F operating 
at $300 \mathrm{kV}$ ). The TEM sample was prepared in cross section and initially thinned using a tripod polishing. Further thinning was done using a Gatan Precision Ion Polisher 691 to achieve electron transparency. Some AZO films were also subjected to rapid thermal annealing (RTA) under $\mathrm{N}_{2}$ ambient in the temperature range of $300-600^{\circ} \mathrm{C}$ for time duration of $10-200 \mathrm{~s}$.

\section{Results and Discussion}

\subsection{Deposition rate}

The deposition rate was determined to be about $0.10 \mathrm{~nm} /$ pulse when the substrate was near room temperature, gradually falling to $0.05 \mathrm{~nm} /$ pulse when increasing the substrate temperature at about $300^{\circ} \mathrm{C}$. No film could be deposited when the substrate temperature exceeded $350^{\circ} \mathrm{C}$. This observation can be explained with re-evaporation and non-sticking of the arriving metal ions. A deposition of $0.1 \mathrm{~nm}$ per pulse is equivalent to a very high instantaneous rate of $0.1 \mathrm{~nm} / 1 \mathrm{~ms}=100 \mathrm{~nm} / \mathrm{s}$. Some of the metal will not react to oxide on the surface during the pulse time but in between pulses. That means that a transient metal film, consisting mostly of zinc, is subject to both evaporation and oxidation, and evaporation becomes significant for the high vapor pressure material zinc as the substrate temperature is increased.

\subsection{Crystal structure}

The AZO films deposited by the filtered cathodic arc method on glass substrates are polycrystalline with preferred c-axis orientation, which is also typical for films obtained by other deposition techniques. X-ray diffraction (XRD) shows only the 0002 and 0004 diffraction peaks in all of the films we studied. Grain size of $\sim 50-150 \mathrm{~nm}$ for film thickness of 50 to $400 \mathrm{~nm}$ are typical for most of the films deposited at $>100^{\circ} \mathrm{C}$. Fig. 2 summarizes the lattice constant and 
grain size for $\mathrm{AZO}$ films grown at $300^{\circ} \mathrm{C}$ as a function of film thickness. The lattice parameter of the AZO films is typically smaller than that of bulk $\mathrm{ZnO}$ crystal. Fig. 2 shows that the grain size of the film increases monotonically with film thickness. The small grain size of $<50 \mathrm{~nm}$ for films $<150 \mathrm{~nm}$ thick will limit the electron mobility in these films due to scattering at the grain boundaries. This is further supported by TEM results mentioned later in this paper.

\subsection{Electrical Properties}

Figure 3 shows the resistivity $(\rho)$, electron concentration $(n)$ and mobility $(\mu)$ of AZO (3 at $\% \mathrm{Al})$ films as a function of substrate temperature during film deposition measured by the Hall effect. Only films with thickness in the range of 50-100 nm are compared in this figure because we were specifically interested in those thin films. One can see that AZO film deposited at room temperature have poor electrical properties and that optimum electrical properties with low resistivity $\rho \sim 5 \times 10^{-4} \Omega \mathrm{cm}$, high carrier concentration $n \sim 5.5 \times 10^{20} \mathrm{~cm}^{-3}$ and high mobility $\mu \sim 18 \mathrm{~cm}^{2} /$ Vs are obtained when the films are grown at about $200^{\circ} \mathrm{C}$.

Including now a set of films deposited with greater thickness, we see in Fig. 4 that the electrical properties of $\mathrm{AZO}$ films grown at $300^{\circ} \mathrm{C}$ improve as the thickness increases; the best parameters are achieved for $\sim 400 \mathrm{~nm}$ thick films with a resistivity as low as $\rho \sim 2 \times 10^{-4} \Omega \mathrm{cm}$, and with a mobility as high as $\mu \sim 40 \mathrm{~cm}^{2} / \mathrm{Vs}$. Considering even thicker films of $500 \mathrm{~nm}$, and aluminum doping concentration of 2 at $\%$ and 6 at $\%$, we see from Fig. 5 that there is again a minimum in resistivity at about $200^{\circ} \mathrm{C}$.

It is interesting to plot the dependence of the mobility on the electron concentration, which is done in Fig. 6 for both thin $(<100 \mathrm{~nm})$ and thick $(>100 \mathrm{~nm})$ films. While no clear dependence can be concluded for thin films $(<100 \mathrm{~nm})$, a monotonic increase of mobility with 
electron concentration is observed for the thick films. Mobility is limited by various scattering mechanisms, and in our case of films made by pulsed cathodic arcs it appears that the conventional ionized impurity scattering mechanism is not dominant because electron mobility does not decrease as the electron concentration increases (e.g. chapter 2 of [7]). Rather, grain boundary scattering seems to be more important. Fig. 7 demonstrates that both the electron concentration and mobility for thick film $>100 \mathrm{~nm}$ increase with grain size (which in turn is related to thickness, see Fig. 2, and substrate temperature).

\subsection{Optical properties}

Not only the electrical but also the optical properties depend greatly on the substrate temperature. Films grown at room temperature are usually absorbing and appear brownish to the eye while films grown at elevated temperature are much more transparent. Using the transmittance curves we can derive Fig. 8 which shows the maximum transmittance, which is found in the wavelength range from $500 \mathrm{~nm}$ to $900 \mathrm{~nm}$, of thin AZO (100 nm, 3 at $\% \mathrm{Al})$ films as a function of growth temperature. We see a steady increase in transmittance with increasing temperature until about $200^{\circ} \mathrm{C}$ is reached.

Figure 9 shows the transmittance as a function of wavelength for much thicker (about 500 nm) AZO films for two different aluminum doping levels grown at $200^{\circ} \mathrm{C}$. High doping levels cause greater carrier concentration and thus a shift of the plasma edge in the near infrared towards shorter wavelengths.

Typically, the band gap $\left(E_{g}\right)$ of a direct gap semiconductor is found from the experimental optical absorption spectra by making a linear extrapolation of the square of the absorption coefficient (or this value multiplied by the square of the photon energy) to the energy 
axis. Fig. 10 shows the square of the absorption coefficient $\alpha$ of two AZO films (70 nm and $400 \mathrm{~nm}$ thick deposited at $\sim 300^{\circ} \mathrm{C}$ ) with different electron concentrations. Linear extrapolations of $\alpha^{2}$ are also displayed as solid lines. As the electron concentration of the film increases, the optical gap also increases (from $\sim 3.5-3.7 \mathrm{eV}$ ) due to the well-known Burstein-Moss shift, which is a blue shift of the absorption edge that occurs as the lower conduction band states become occupied and thus unavailable for carrier excitation.

\subsection{Effects of Rapid Thermal Annealing}

So far, the best electrical properties were obtained at growth temperatures of about $200^{\circ} \mathrm{C}$, and higher temperatures generally lead to films of improved optical properties. Rapid thermal annealing (RTA) could be an interesting route for further improving the optical and electrical performance due to grain growth and the removal of defects [30, 31].

The effect of RTA was first studied on samples deposited at room temperature (RT); RTA was done in nitrogen atmosphere in steps lasting 10 seconds each, and films were allowed to cool down to RT between steps for taking the measurements. Fig. 11 shows that even as the properties greatly improve upon RTA, films deposited at room temperature do not match the performance of films grown at elevated temperature. The choice of 10 seconds annealing time was based on another test series where different annealing times between $10 \mathrm{~s}$ and $200 \mathrm{~s}$ have been tried: the longer time did not result in any further significant improvement.

Since our special interest is in very thin films $(<100 \mathrm{~nm})$, RTA was applied to a $64 \mathrm{~nm}$ film deposited at $200^{\circ} \mathrm{C}$. Cutting the substrate in pieces and applying different RTA times to them showed that $10 \mathrm{~s}$ RTA to $450^{\circ} \mathrm{C}$ improves mobility, though longer heat treatment is detrimental (Fig. 12). 


\subsection{Transmission electron microscopy}

Transmission electron microscopy (TEM) analysis was carried out for a sample which was deposited using a 2 at $\%$ Al-doped $\mathrm{Zn}$ cathode at a nominal growth temperature of $95^{\circ} \mathrm{C}$. The actual substrate temperature was about $88^{\circ} \mathrm{C}$ at the beginning of the deposition process and increased to $102^{\circ} \mathrm{C}$ due to heating by the deposition process. The main features in the brightfield image indicated in Fig. 13(a) are (i) the epoxy glue, (ii) the Al-doped $\mathrm{ZnO}$ film and (iii) the $\mathrm{SiO}_{2}$ substrate. The electron diffraction pattern of this region shows crystal grains orientated with (002) planes parallel to the surface. The darkfield image, Fig. 13(b), from the (002) reflection shows a high density of defects; the thin film consists of small crystals which are extended throughout the thickness of the film. The high resolution image in Fig. 14 clearly shows small crystals of 10-15 $\mathrm{nm}$ in size closely spaced together. Having such a high concentration of defects is likely to increase the scattering at grain boundaries therefore lowering the mobility for films grown at this relatively low temperature.

\subsection{Figure of merit}

A figure of merit for comparing different films can be defined in various ways, each definition giving certain relative weights to the important parameters, here transmittance and resistivity. For example, one could look at the ratio of average visible transmittance, $T_{\text {vis }}$, and resistivity, $\rho,[32,33]$

$$
f_{\text {vis }}=T_{\text {vis }} / \rho
$$

though this leads to a preference of very thin films. A more reasonable ratio is [34]

$$
F_{\text {vis }}=T_{\text {vis }} / R_{s},
$$


where $R_{s}=\rho d$ is the sheet resistance of the film of thickness $d$. However, as argued by Haacke [35], such definition implies the conclusion that films with transmittance $\sim 30-40 \%$ are optimal, which clearly is too low for most applications. Therefore, the modified figure of merit [35]

$$
\phi_{v i s}=T_{v i s}^{10} / R_{s}
$$

appears to be a much better choice. Each of the films can be evaluated; Fig. 15 shows an example of AZO sample sets with doping levels of 2 at $\% \mathrm{Al}$ and 6 at $\% \mathrm{Al}$. The figure of merit allows us to quantify film quality and confirm that a growth temperature of about $200^{\circ} \mathrm{C}$ results in the best films, and this optimum temperature seems to be relatively independent of the doping level.

\section{Summary and Conclusions}

A number of different sample sets of aluminum doped zinc oxide were synthesized with pulsed, filtered cathodic arc deposition. This technique was chosen with the goal to obtain high quality material that does not exhibit the damage inflicted by high energy negative oxygen ions, which are known to occur with magnetron sputtering. The number of process variables is large, and therefore some emphasis was put on the effect of the aluminum doping level, substrate temperature, film thickness, and rapid annealing temperature and time. It was found, similar to other deposition methods, that the resistivity decreases with increasing substrate temperature and film thickness. More specifically, the improvement can be traced to an increase in mobility with substrate temperature and to an increase in both carrier concentration and mobility when the film thickness is increased. The perhaps most remarkable result is that the resistivity can be in the $10^{-4} \Omega \mathrm{cm}$ region even for films as thin as $60 \mathrm{~nm}$. The growth (substrate) temperature emerged 
as the most influential parameter: judging by the figure of merit $\phi_{v i s}=T_{v i s}^{10} / R_{s}$, where $T_{v i s}$ is the average visible transmittance and $R_{s}$ is the sheet resistance, the best films were deposited at about $200^{\circ} \mathrm{C}$. Somewhat higher temperatures, up to $300^{\circ} \mathrm{C}$, also resulted in very good films, especially for thicker films, with the lowest resistivity being $2 \times 10^{-4} \Omega \mathrm{cm}$ and the high mobility exceeding $40 \mathrm{~cm}^{2} / \mathrm{Vs}$.

Rapid thermal annealing greatly improves the properties of room temperature films but their final properties do not match those of films grown at elevated temperature. RTA of films grown at higher temperature may lead to a slight increase in mobility and a related decrease in resistivity; yet the most significant improvement was observed for thin $(<100 \mathrm{~nm})$ films when the annealing time was only $10 \mathrm{~s}$.

Grain boundary scattering, as opposed to impurity scattering, is suggested as the dominant mechanism limiting electron mobility because the mobility increases when plotted against the electron concentration.

We conclude that pulsed filtered arc deposition is a technology suitable to make high quality AZO material, especially when the interest is in thin $(<100 \mathrm{~nm})$ films. The range of assisting energy seems to be close to optimum, and the obtained optical and electrical parameters are comparable to or slightly better than the best films obtained other methods such as sputtering and pulsed laser deposition $[7,17,36]$. Since they do not much exceed those best results, one may suspect that there are indeed fundamental limits to the material $[9,37,38]$ and/or that the highest energy in the zinc ion energy distribution $(\sim 50 \mathrm{eV})$ has a detrimental influence on film growth.

\section{Acknowledgments}


This work was supported by the Assistant Secretary for Energy Efficiency and Renewable Energy, Office of Building Technology, and by the Director, Office of Science, Office of Basic Energy Sciences, Division of Materials Sciences and Engineering of the U.S. Department of Energy under Contract No. DE-AC02-05CH11231. K.M. Yu thanks RoseStreet Labs Energy, Inc. for additional support; S.H.N. Lim gratefully acknowledges the financial support by the Australian Research Council (ARC project DP0666883) and the Australian Institute of Nuclear Science and Engineering (AINSE grants AINGRA07012P and AINGRA07094); J. Rosén and J. Andersson acknowledge support by the SSF Strategic Research Centre on Materials Science for Nanoscale Surface Engineering, and the Wenner-Gren Foundations (both Sweden), respectively. 


\section{References}

[1] I. Hamberg, C.G. Granqvist, J. Appl. Phys. 60 (1986) R123.

[2] B.E. Sernelius, K.F. Berggren, Z.C. Jin, I. Hamberg, C.G. Granqvist, Phys. Rev. B 37 (1988) 10244.

[3] B. Szyszka, Thin Solid Films 351 (1999) 164.

[4] C.G. Granqvist, Solar Energy Materials and Solar Cells 91 (2007) 1529.

[5] A. Walsh, J.L.F. Da Silva, S.-H. Wei, Phys. Rev. B 78 (2008) 075211.

[6] T. Minami, Thin Solid Films 516 (2008) 5822.

[7] K. Ellmer, A. Klein, B. Rech (Eds.), Transparent Conductive Zinc Oxide: Basics and Applications in Thin Film Solar Cells, Springer, New York, 2008.

[8] F. Ruske, A. Pflug, V. Sittinger, B. Szyszka, D. Greiner, B. Rech, Thin Solid Films In Press, Accepted Manuscript (2009).

[9] K. Ellmer, J. Phys. D: Appl. Phys. 34 (2001) 3097.

[10] S. Goldsmith, Surf. Coat. Technol. 201 (2006) 3993.

[11] S. Mráz, J.M. Schneider, J. Appl. Phys. 100 (2006) 023503.

[12] X.B. Zhang, Z.L. Pei, J. Gong, C. Sun, J. Appl. Phys. 101 (2007) 014910.

[13] T. Minami, T. Yamamoto, T. Miyata, Thin Solid Films 366 (2000) 63.

[14] S. Mráz, J.M. Schneider, Appl. Phys. Lett. 89 (2006) 051502.

[15] H. Gnaser, in: R. Behrisch, W. Eckstein (Eds.), Sputtering by Particle Bombardment, Springer, Berlin, 2007, p. 231.

[16] A. Anders, Cathodic Arcs: From Fractal Spots to Energetic Condensation, Springer, New York, 2008. 
[17] U. Özgür, Y.I. Alivov, C. Liu, A. Teke, M.A. Reshchikov, S. Dogan, V. Avrutin, S.J.

Cho, H. Morkoc, J. Appl. Phys. 98 (2005) 041301.

[18] A. Anders, G.Y. Yushkov, J. Appl. Phys. 91 (2002) 4824.

[19] E. Byon, A. Anders, J. Appl. Phys. 93 (2003) 1899.

[20] J. Rosén, A. Anders, L. Hultman, J.M. Schneider, J. Appl. Phys. 94 (2003) 1414.

[21] J. Rosén, A. Anders, S. Mráz, A. Atiser, J.M. Schneider, J. Appl. Phys. 99 (2006) 123303.

[22] A. Anders, Vacuum 67 (2002) 673.

[23] J.S. Colligon, J. Vac. Sci. Technol. A 13 (1995) 1649.

[24] O.R. Monteiro, Annual Rev. Mat. Sci. 31 (2001) 111.

[25] A. Anders, Appl. Phys. Lett. 80 (2002) 1100.

[26] T. David, S. Goldsmith, R.L. Boxman, J. Phys. D: Appl. Phys. 38 (2005) 2407.

[27] Y.G. Wang, S.P. Lau, H.W. Lee, S.F. Yu, B.K. Tay, X.H. Zhang, K.Y. Tse, H.H. Hng, J. Appl. Phys. 94 (2003) 1597.

[28] R.A. MacGill, M.R. Dickinson, A. Anders, O.R. Monteiro, I.G. Brown, Rev. Sci. Instrum. 69/2 (1998) 801.

[29] A. Anders, R.A. MacGill, T.A. McVeigh, Rev. Sci. Instrum. 70 (1999) 4532.

[30] T. Yen, D. Strome, S.J. Kim, A. Cartwright, W.A. Anderson, J. Electronic Materials 37 (2008) 764 .

[31] Y.-C. Lee, S.-Y. Hu, W. Water, K.-K. Tiong, Z.-C. Feng, Y.-T. Chen, J.-C. Huang, J.-W. Lee, C.-C. Huang, J.-L. Shen, M.-H. Cheng, J. Luminescence 129 (2009) 148.

[32] C. Terrier, J.P. Chatelon, J.A. Roger, Thin Solid Films 295 (1997) 95. 
[33] H.M. Ali, H.A. Mohamed, M.M. Wakkad, M.F. Hasaneen, Thin Solid Films 515 (2007) 3024.

[34] D.B. Fraser, H.D. Cook, J. Electrochem. Soc. 119 (1972) 1368.

[35] G. Haacke, J. Appl. Phys. 47 (1976) 4086.

[36] M. Kon, P.K. Song, Y. Shigesato, P. Frach, A. Mizukami, K. Suzuki, Jpn. J. Appl. Phys. 41/Part 1 (2002) 814.

[37] R. Cebulla, R. Wendt, K. Ellmer, J. Appl. Phys. 83 (1998) 1087.

[38] K. Ellmer, J. Phys. D: Appl. Phys. 33 (2000) R17. 


\section{Figure Captions}

Fig. 1 Setup of filtered arc deposition system: the zinc-aluminum arc plasma is freed from most macroparticles by a 90-degree open filter coil; the substrate is rotated for improved uniformity; heating of its front side is done by a radiative heater.

Fig. 2 XRD measurements giving the lattice parameter, c, and the average grain size of AZO films grown at $300^{\circ} \mathrm{C}$ substrate temperature, as a function of film thickness.

Fig. 3 Carrier concentration, mobility, and film resistivity for thin $(<100 \mathrm{~nm})$ AZO (3 at $\% \mathrm{Al})$ films as a function of growth temperature.

Fig. 4 Carrier concentration, mobility, and film resistivity of AZO films as a function of film thickness.

Fig. 5 Resistivity of 2 at $\%$ and 6 at $\%$ Al-doped $\mathrm{ZnO}$ films of $500 \mathrm{~nm}$ thickness as function of growth temperature.

Fig. 6 Mobility of both thin $(<100 \mathrm{~nm})$ and thick $(>100 \mathrm{~nm})$ AZO films (3 at\% Al) plotted as a function of electron concentration.

Fig. 7 Electron concentration and mobility of AZO films as a function of grain size.

Fig. 8 Maximum transmittance of thin AZO (100 nm, 3 at\% Al) in the spectral range 500-900 $\mathrm{nm}$ as a function of growth temperature.

Fig. 9 Transmittance as a function of wavelength for about $500 \mathrm{~nm}$ thick AZO films grown at $200^{\circ} \mathrm{C}$ for two different aluminum doping levels.

Fig. 10 Square of the absorption coefficient $\alpha$ of $70 \mathrm{~nm}$ and $400 \mathrm{~nm}$ thick AZO films, both deposited at $\sim 300^{\circ} \mathrm{C}$; the $70 \mathrm{~nm}$ film is the one with lower electron concentrations. Linear extrapolations of $\alpha^{2}$ show that AZO is a direct gap semiconductor with the optical gap dependent on the electron concentration. 
Fig. 11 Rapid thermal annealing of AZO films (3 at\%) deposited at room temperature; each annealing step lasted $10 \mathrm{~s}$, the sample was cooled to room temperature for measurements, followed by the next RTA step.

Fig. 12 Effect of the RTA time on electrical properties of a $64 \mathrm{~nm} \mathrm{AZO} \mathrm{(3} \mathrm{at \%} \mathrm{Al).}$

Fig. 13 TEM brightfield image (a) and darkfield image (b) of an AZO (3 at\%) film grown at nominal $95^{\circ} \mathrm{C}$ on $\mathrm{SiO}_{2}$; (i) is the epoxy glue used in preparing the sample, (ii) is the $\mathrm{AZO}$ film, and (iii) is the $\mathrm{SiO}_{2}$ substrate.

Fig. 14 High resolution TEM of an AZO film $(2 \mathrm{at} \% \mathrm{Al})$ grown at nominal $95^{\circ} \mathrm{C}$ : one can see a high density of defects and numerous small grains of typically $10 \mathrm{~nm}$ in size, most of them oriented along the c-axis (growth direction).

Fig. 15 Figure of merit, as defined by equation (3), for various films with 2 at $\%$ and 6 at $\%$ aluminum doping, as a function of the growth temperature. 


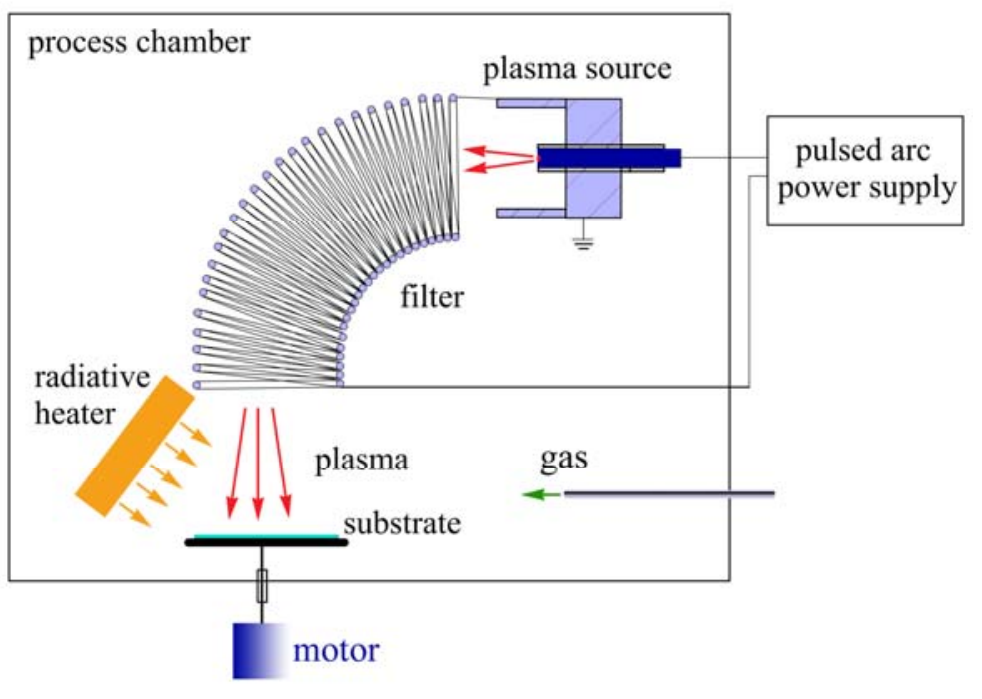

Fig. 1 


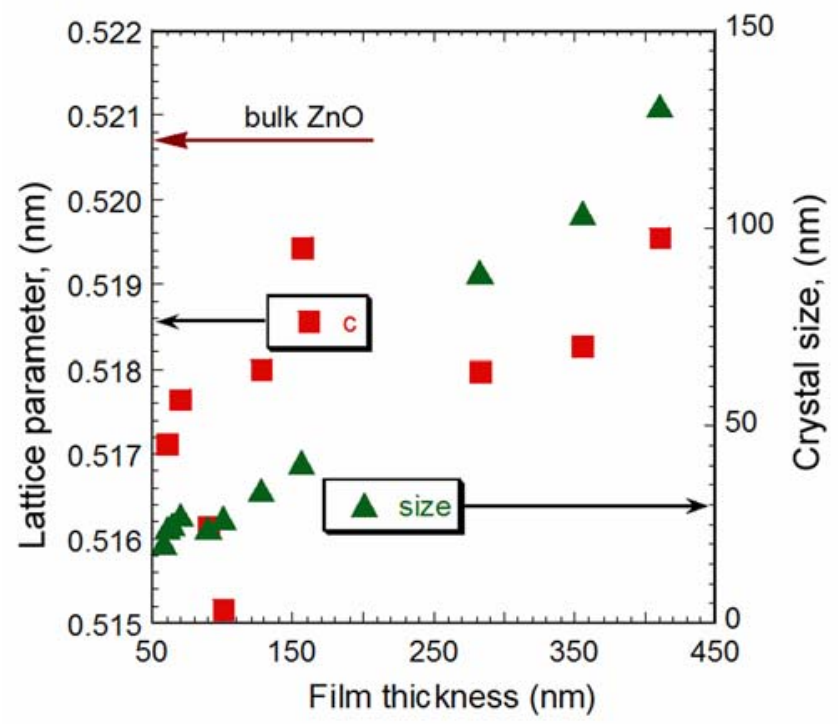

Fig. 2 


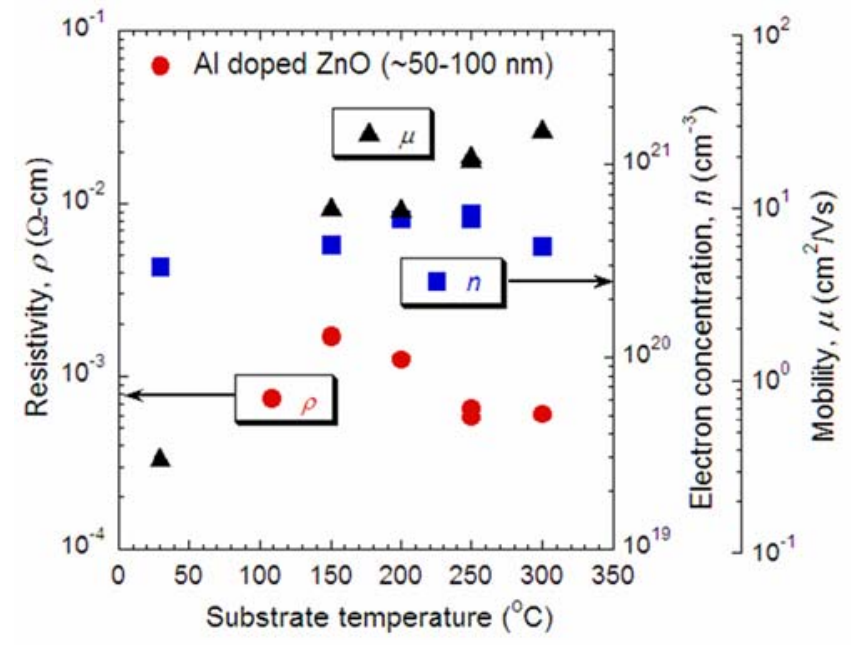

Fig. 3 


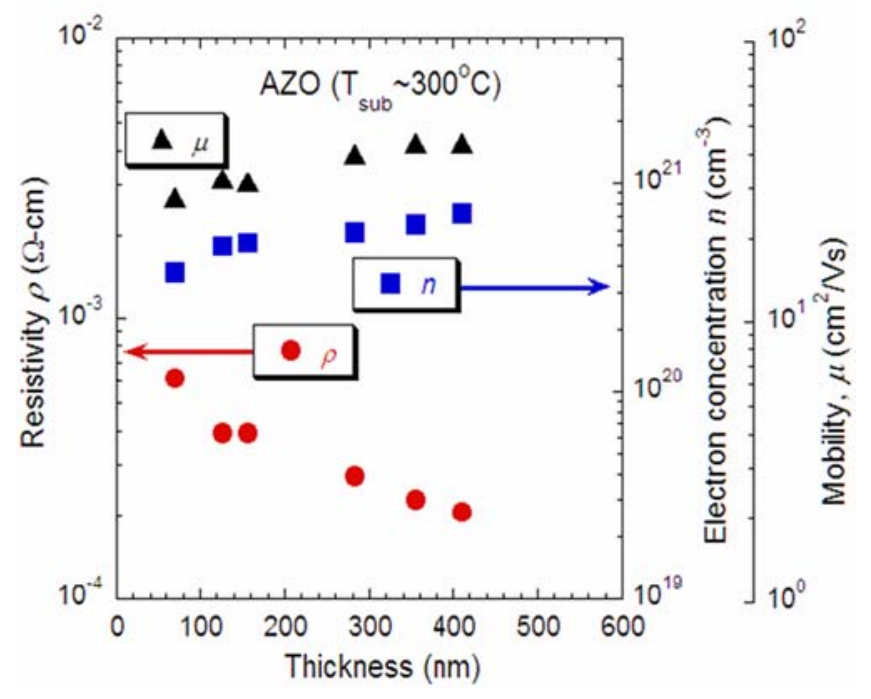

Fig. 4 


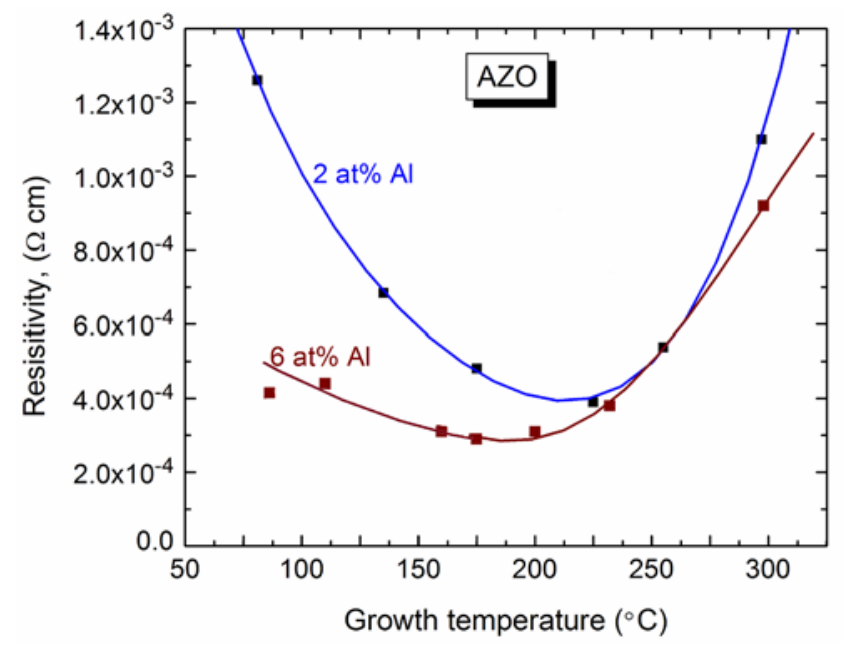

Fig. 5 


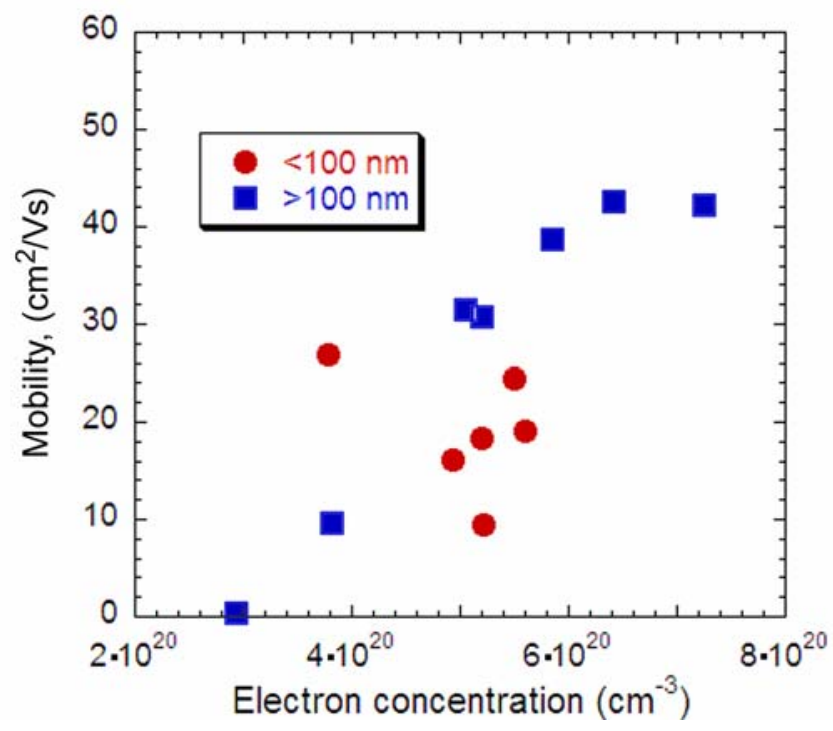

Fig. 6 


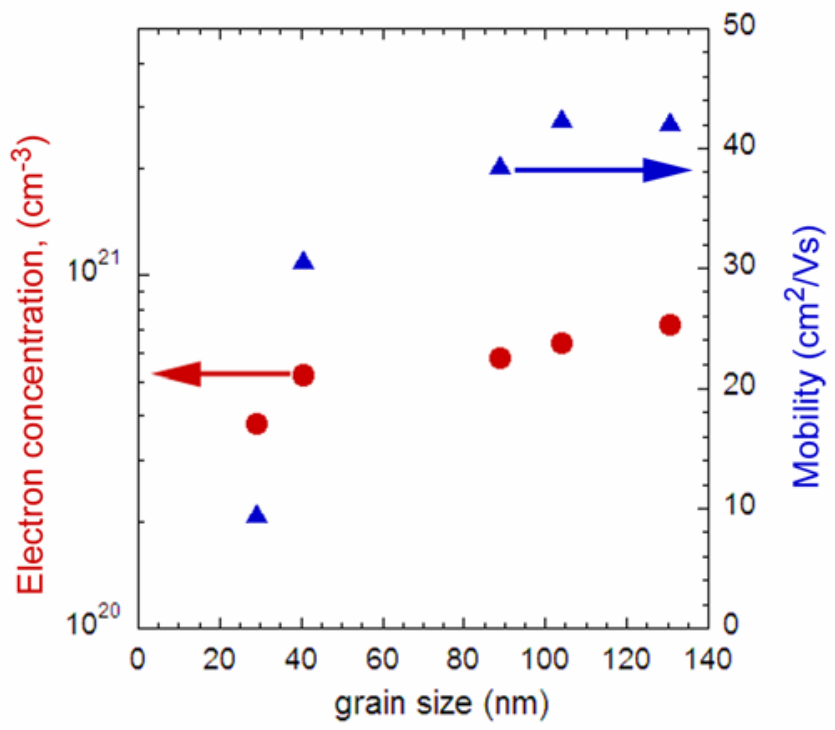

Fig. 7 


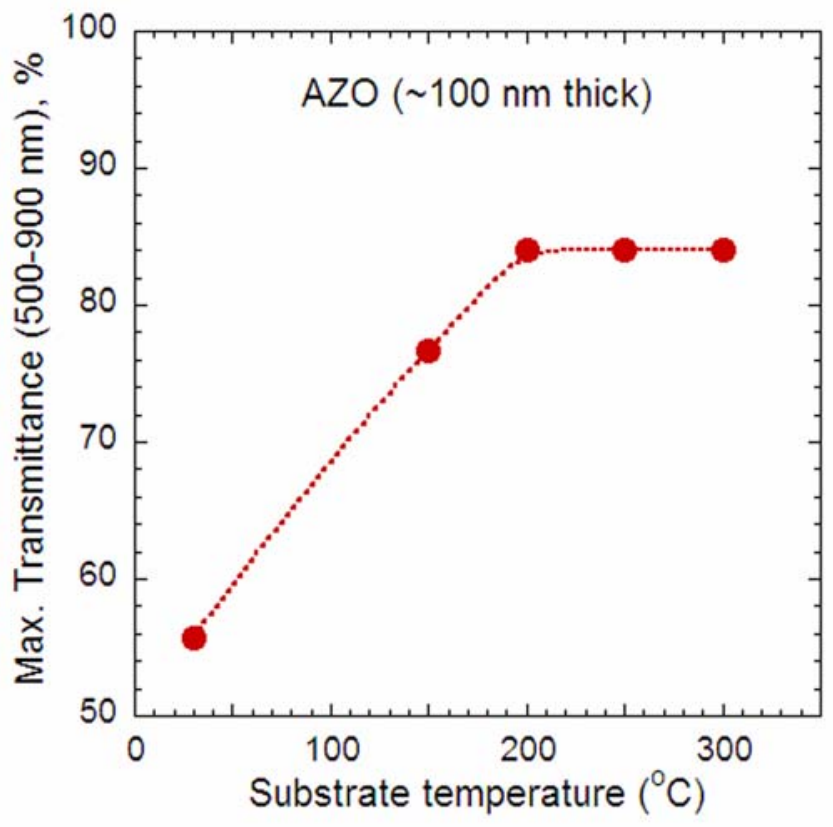

Fig. 8 


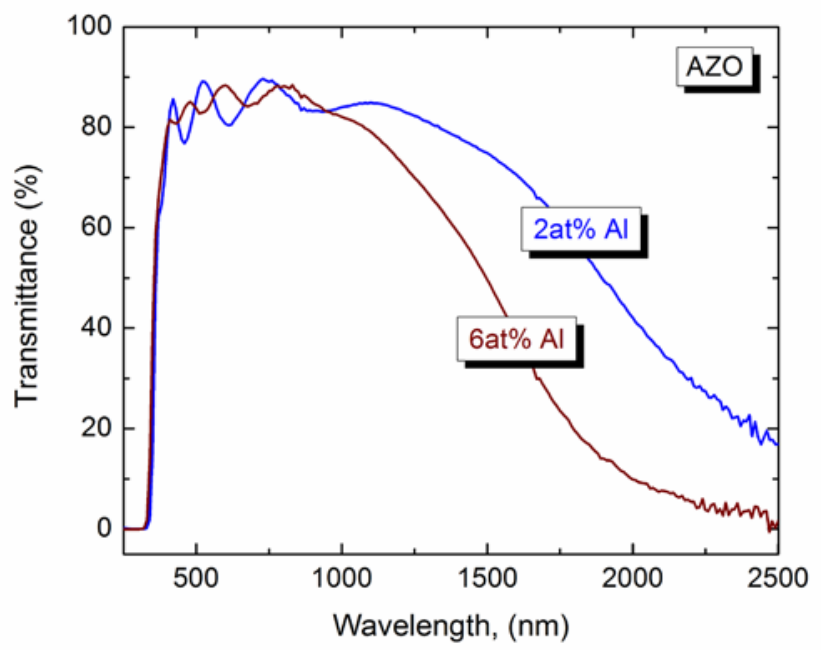

Fig. 9 


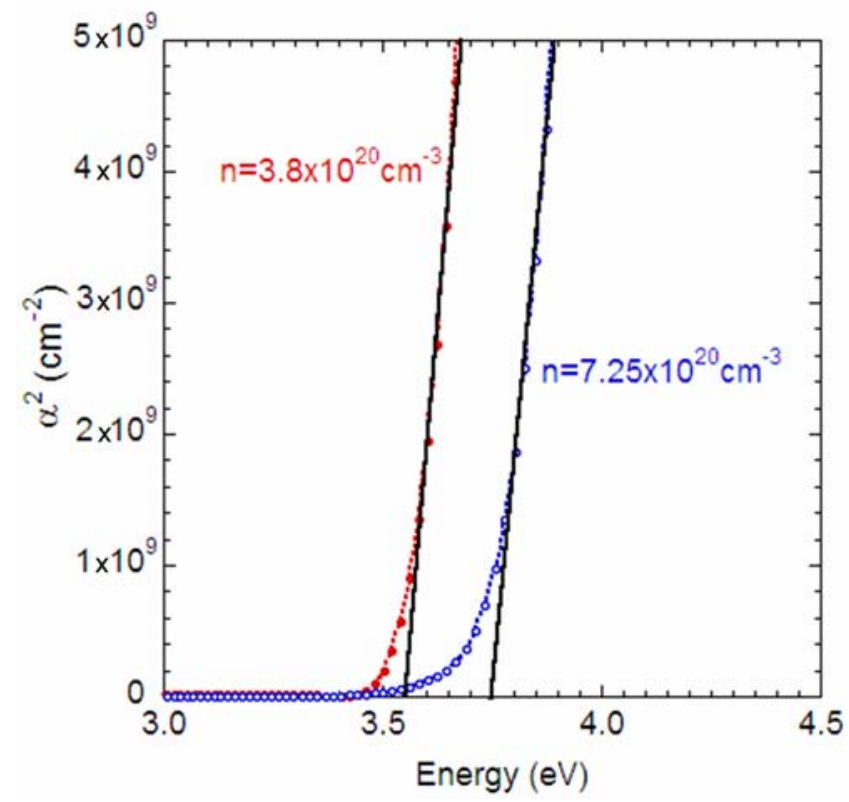

Fig. 10 

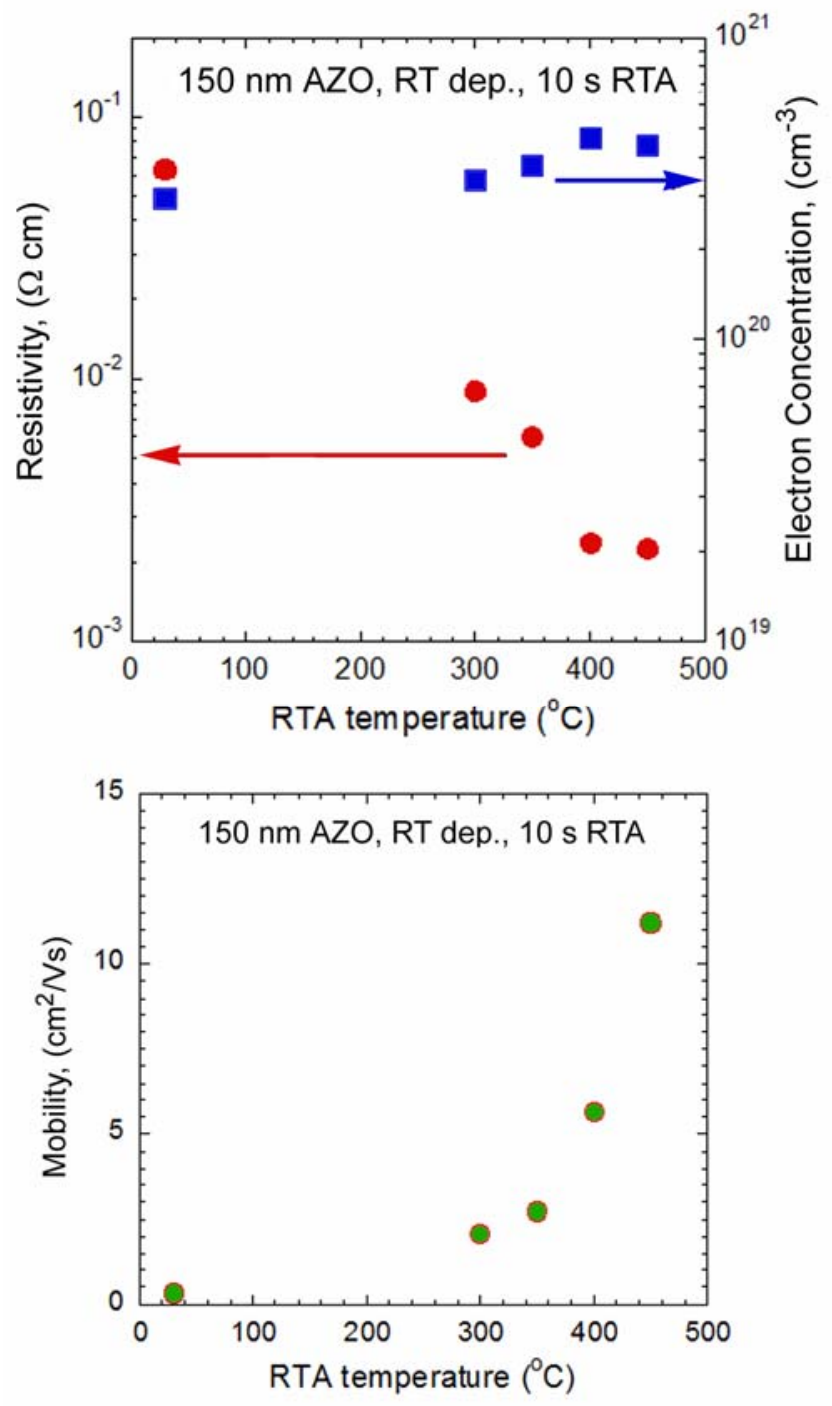

Fig. 11 

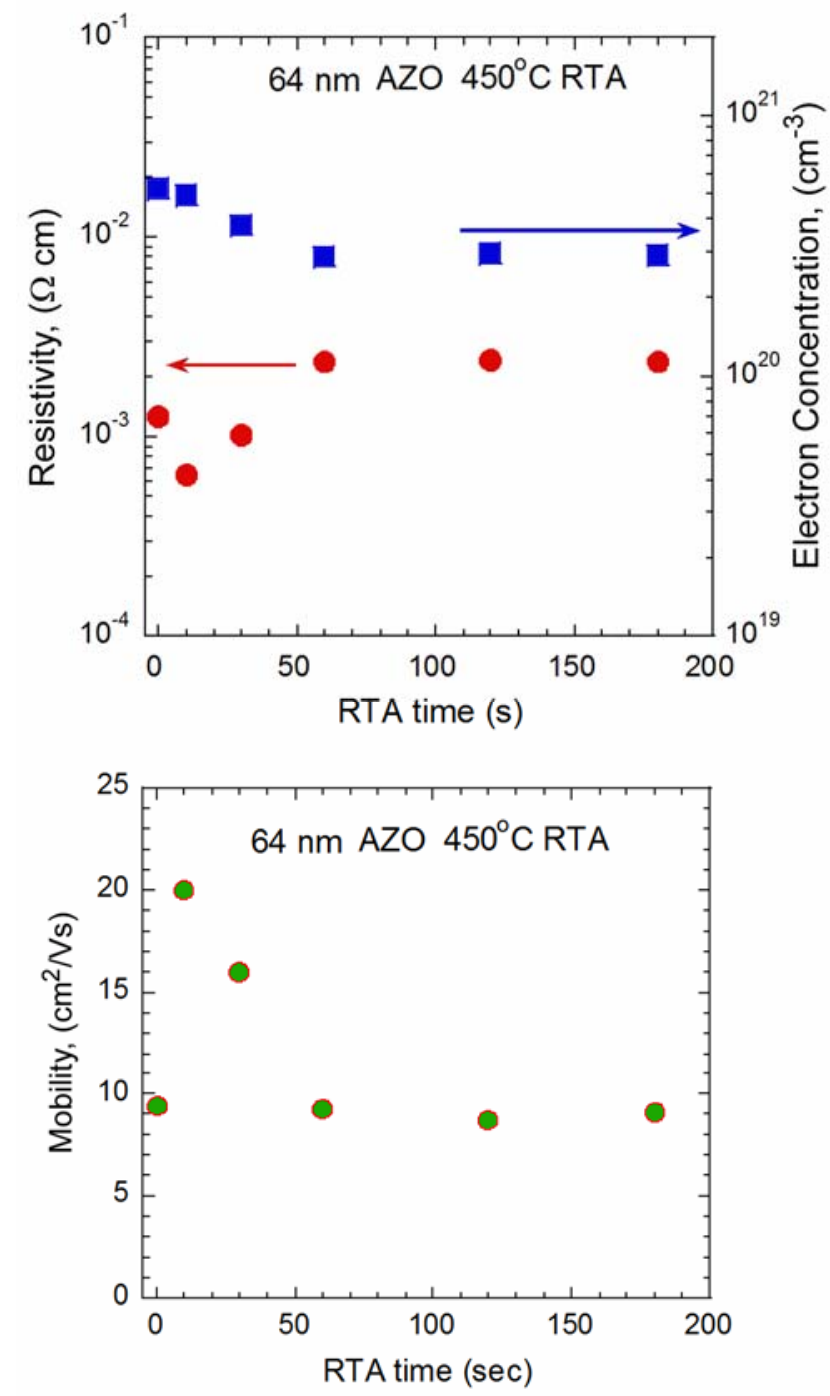

Fig. 12 


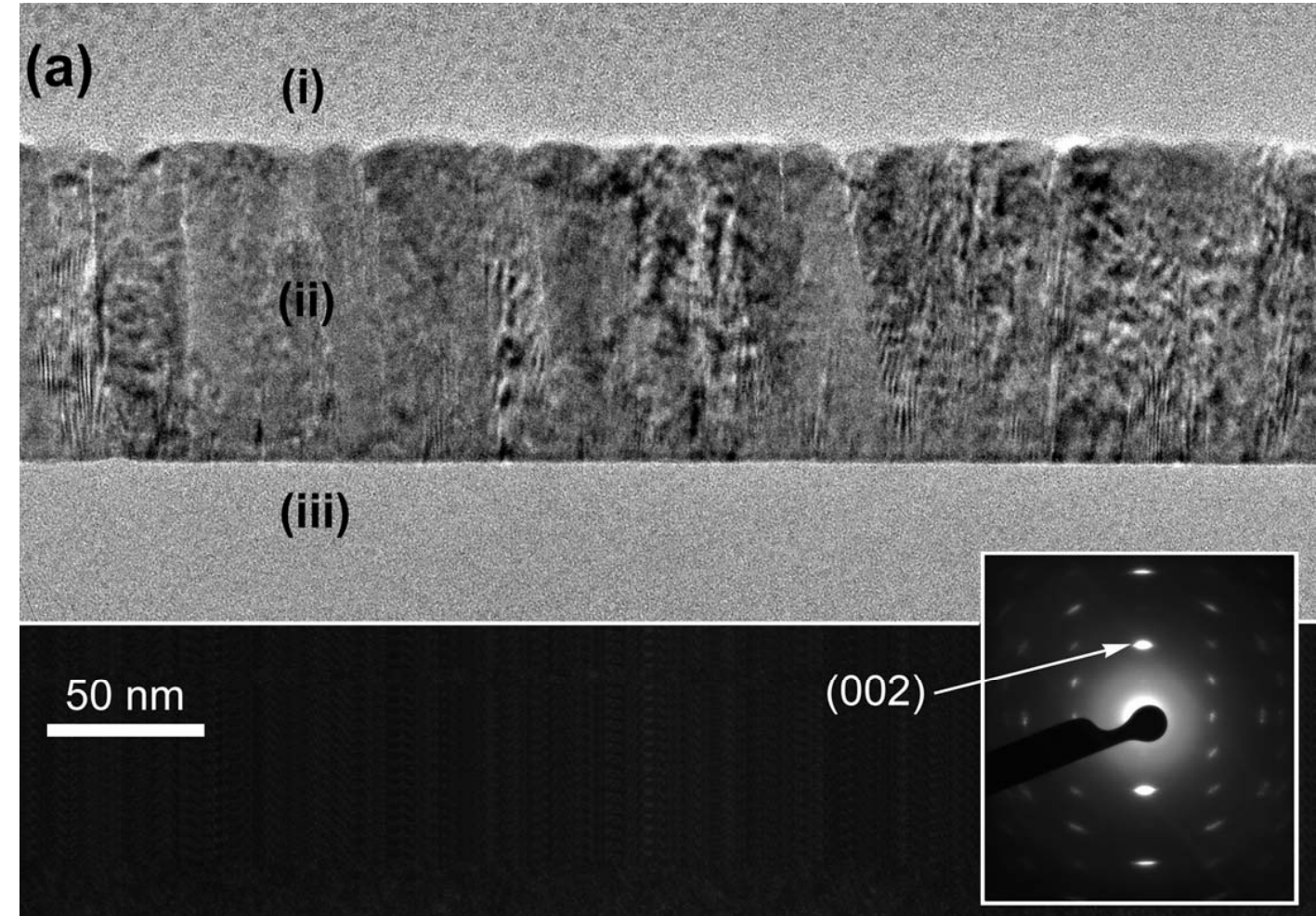

(b)

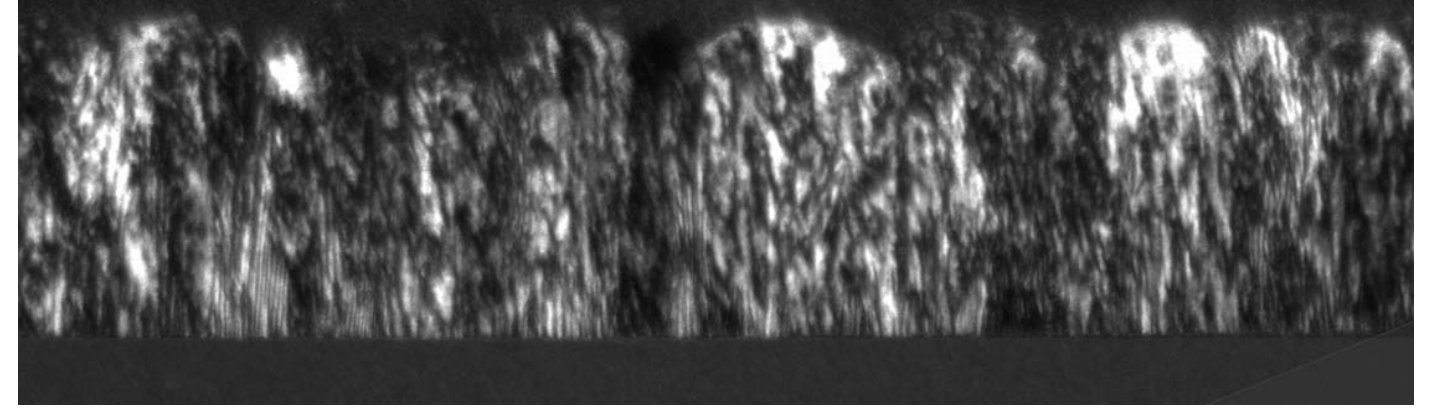

Fig.13 


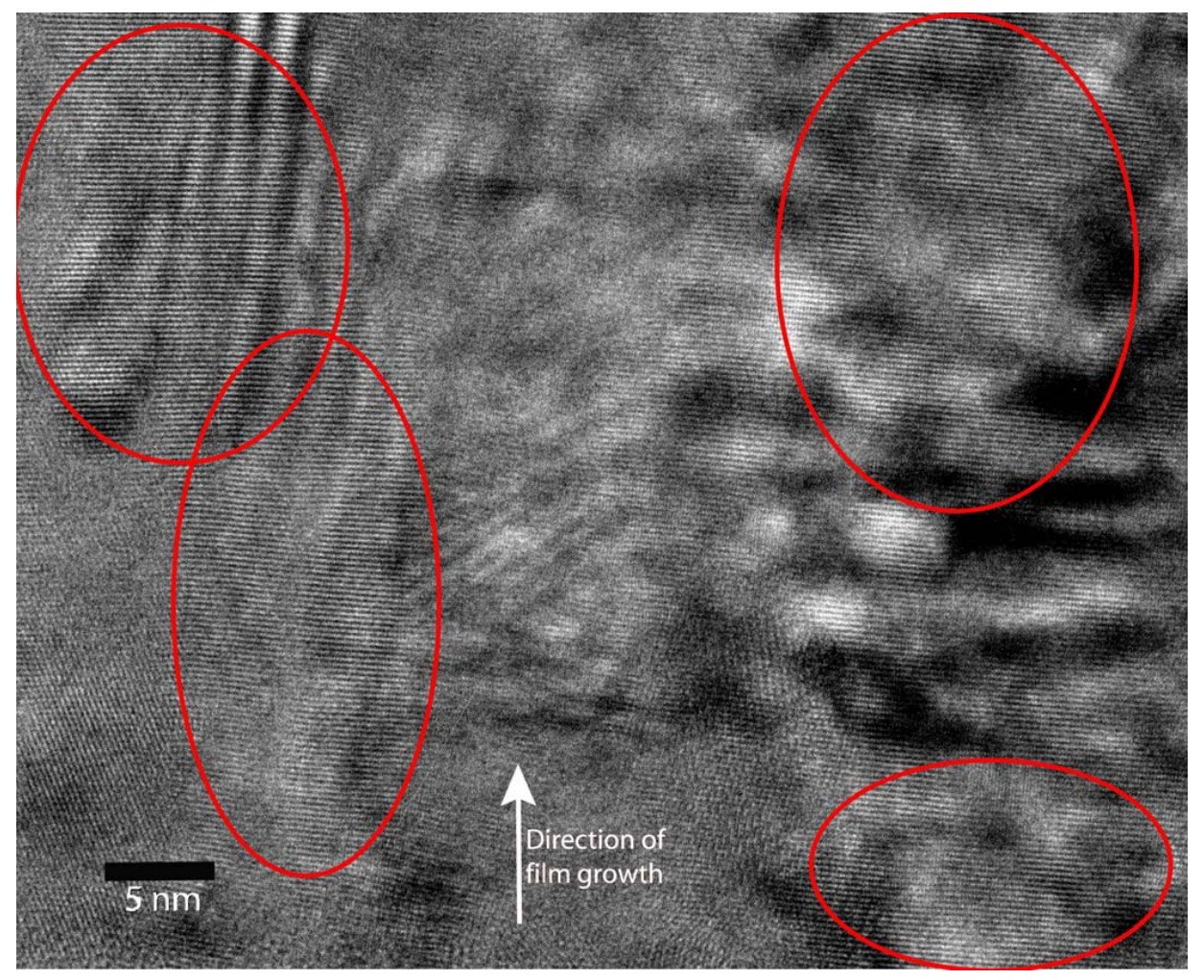

Fig. 14 


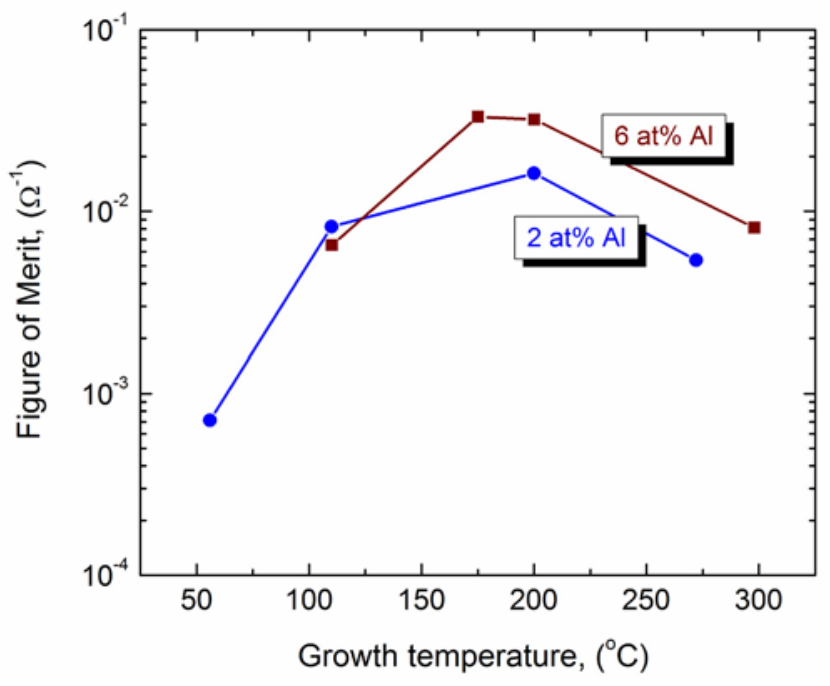

Fig. 15 